\title{
The current density order based on the Ginzburg-Landau description
}

\author{
Z. Bousnane ${ }^{1}$, N. Merabtine ${ }^{2}$, M. Benslama ${ }^{2}$, F. Bousaad ${ }^{1}$ \\ ${ }^{1}$ Physics Department, Faculty of Science, University of Batna, 05000 Algeria \\ ${ }^{2}$ Electromagnetism and Telecommunication Laboratory, Electronics Department, \\ Faculty of Engineering, University of Constantine, 25000 Algeria \\ Corresponding author: malekbenslama@hotmail.com
}

\begin{abstract}
The goal of this survey is to deduce the grandeurs, or the set of grandeurs, from which is derived simultaneously as a linear combination of densities of states, current density matrix and the reduced entropy, according to the general fact that the logarithm of the distribution is additive first integral. In this perspective, we introduce the notations $\hat{\mathrm{I}}|\Psi|^{2}+\hat{\mathrm{J}} \mathrm{j}_{\alpha \beta}+\hat{\mathrm{K}} \mathrm{S}_{\alpha \beta}$, which gives to the logarithm of the distribution as the quaternionic picture of the operatorial transcriptions, this must follow the behaviour of a canonical distribution through the interval of the transitions. It seems that the nonreproducibility is caused essentially by the fact of absolute separability of dimensions between the observed and observer. The reduced entropy will suggest the inner displaying of observer, the invariance of unsymmetric order parameter products will be an expression of reproducibility. We must have a displaying of such products over inner dimensions, allowing to translate a limit of the displaying of stationary levels of macroscopic bodies over inner distances. $\hat{I}$ is the parity operator and will act under respect or violation of products as uncertainties, $\hat{\mathrm{J}}$ is representing measurement process decomposing layers, sublayers and orbitals according to the thresholds logics answering how cold will be felt to transgress the conventional univoc filling rules, $\hat{\mathrm{K}}$ represents measurement process realising the centesimal entropy depth penetration. The introduction of such notations will be justified by the fact that the $\rho$-distribution, introduced as an unsymmetric product of order parameters, is defined per pavement pavement as defined by J.H. Poincaré.
\end{abstract}

Keywords: current density order, pavement, superconductivity.

Manuscript received 21.02.06; accepted for publication 26.03.07; published online 01.06.07.

\section{Introduction}

The reduced entropy defining the eigenvalue is equivalent to say that the possible order parameters describing the superconductor state are recursive, the recursivity of the order parameter implies that among the whole micropossible configurations, over which displayed are the macropossible configurations; there exist configurations that seems to be displayed over an effective subset of configurations, thus the symmetry order in nature will be as follows:

a) the real symmetries corresponding to the attitude of matter to the order; b) the nonreal symmetries corresponding to the possibilities let by this order to the space to accept the same order.

These considerations above will allow us to realise limitations imposed to the GL description validity.

\section{Distribution law}

As known in the statistical mechanics methods [1], and conformably to the natural character of grandeurs during a phase transitions

$$
\ln _{\alpha}\left(\ln _{\beta} \rho\right)=\hat{\mathrm{I}}|\Psi|^{2}+\ln _{\alpha}\left(\ln _{\beta} \Delta \Gamma\right)-\operatorname{div}_{\alpha} \operatorname{div}_{\beta} j_{\alpha \beta}
$$


with $\frac{d}{d t}|\Psi|^{2}=-\operatorname{div}_{\alpha} \operatorname{div}_{\beta} j_{\alpha \beta}$ is the effective continuity equation. The distribution function $\Delta W$ is given by

$$
\rho(2 \pi \hbar)^{\mathrm{s}} \Delta \Gamma
$$

that gives the number of the macropossible states. Equation (1) is written

$\ln _{\alpha}\left(\ln _{\beta} \rho\right)-\ln _{\alpha}\left(\ln _{\beta} \Delta \Gamma\right)=\hat{I}\left|\Psi^{2}\right|-\operatorname{div}_{\alpha} \operatorname{div}_{\beta} j_{\alpha \beta}$,

and for the $W$ function, we have

$$
\begin{aligned}
& \ln _{\alpha}\left(\ln _{\beta} \frac{\Delta W}{(2 \pi \hbar)^{s}}\left(\Delta \Gamma^{\prime}\right)\right)-\ln _{\alpha}\left(\ln _{\beta} \Delta \Gamma\right)= \\
& =\hat{\mathrm{I}}\left|\Psi^{2}\right|-\operatorname{div}_{\alpha} \operatorname{div}_{\beta} j_{\alpha \beta} .
\end{aligned}
$$

For the definition of the $W$ function, we must have $\frac{1}{\Delta \Gamma \Delta \Gamma^{\prime}} \sim(2 \pi \hbar)^{\mathrm{s}}$ that stipulate an uncertainty principle as follows:

$$
\frac{1}{\Delta \Gamma \Delta \Gamma^{\prime}} \sim \frac{1}{\hbar^{\mathrm{s}}} \text {. }
$$

The recursivity here permits the stability for $\Delta \Gamma$ and $\Delta \Gamma^{\prime}$, and we hear by this an interaction between micropossible configurations, permitting the emergence of homologous one, which having amplitudes and phases linearly homologous between them, which is equivalent to the interaction of micropossible configurations for emerging a news proportional to their images. When the macropossible configurations follow the same processes, this leads to the reproducibility.

Taking $\Delta \Gamma \Delta \Gamma^{\prime} \sim \frac{1}{\hbar^{\mathrm{s}}}$ into account, equation (4) will be written

$$
\ln _{\alpha}\left(\ln _{\beta} \Delta \mathrm{W}\right)=\hat{\mathrm{I}}\left|\Psi^{2}\right|-\operatorname{div}_{\alpha} \operatorname{div}_{\beta} j_{\alpha \beta} .
$$
Eq. (5).

The emergence of recursivity will be generated by

$\Delta \Gamma$ and $\Delta \Gamma^{\prime}$ are the measurement results as a state numbers, which here are a conventional choice, if $\Delta \Gamma$ presents the states number of macropossible informing on real symmetries, $\Delta \Gamma^{\prime}$ is linked to the recursive one.

We write $S_{\alpha \beta}$ as $\ln \Delta \Gamma_{\alpha} \Delta \Gamma_{\beta}^{\prime}$ with

$S_{0}=\overline{S_{\alpha \beta}}$,

which expresses the conservation law of the reduced entropy mean value. If this law is expressed as a function of one statistical weight, we have
$S_{0}=\overline{S_{\alpha \beta}}=-\ln _{\alpha}\left(\ln _{\beta} \Delta \Gamma\right)$.

The normalized $S_{\alpha \beta}$ is written as $S_{\alpha \beta}=\frac{1}{2} \ln \Delta \Gamma_{\alpha} \Delta \Gamma_{\beta}$, on the other hand, we have $S_{\alpha \beta}=$ $k \ln _{\alpha}\left(k \Delta \Gamma_{\beta}\right)$, and $S_{\alpha \beta}=\ln \Delta \Gamma_{\alpha} \Delta \Gamma_{\beta}$ must obey the physical equivalence, we then write

$$
S_{\alpha \beta}=\frac{1}{2}\left[\ln \Delta \Gamma_{\alpha} \Delta \Gamma_{\beta}+\mathrm{k} \ln _{\alpha}\left(\mathrm{k} \ln _{\beta} \Delta \Gamma\right)\right] .
$$

When $S_{\alpha \beta}=S_{0}=0$, we have $\ln \Delta \Gamma_{\alpha} \Delta \Gamma_{\beta}=$ $=-k \ln _{\alpha}\left(k \ln _{\beta} \Delta \Gamma\right)$.

The reduced entropy that we introduce takes the meaning that the natural increasing of entropy through an interval of states is "suffocating" by the dual increasing of entropy through an other interval of states.

The ancient entropy was free, it was in accord with homogenous, isotropic space and uniform time.

The limitations imposed to the entropy in our case are caused by the recursive character of the wave function generating the GL order parameter.

\section{Description}

With the decreasing of temperature, the entropy of the two "candidates" wave functions will be represented as two recursive lens, making the action of "cold" as a reduced entropy.

The cold as a classical being, it was considered, had generated the order parameter with asymptotic form that was a function of the coherence length

$\Psi \sim \eta(a, b) e^{i \frac{S}{\xi(T)}}$,

where $a$ and $b$ are the Landau phenomenological parameters. $\xi(T)$, the Landau coherence length, and $\mathrm{S}$ is the entropy linked to the free energy density by relations:

$F=E-T S(N)=E-\ln \Delta|\Psi|^{2}$,

$F=E-T S(\varphi)=E-\ln \Delta|\Psi|^{2}$,

$F=E-T S(\varphi, N)=E-\ln \Delta\left|\begin{array}{cc}\Delta \Psi & \nabla \Psi^{*} \\ \nabla \Psi & \Delta \Psi^{*}\end{array}\right|$,

where

$S(N)$ is written in N-representation,

$S(\varphi)$ is written in $\varphi$-representation, and

$S(\varphi, N)$ is acting operator bi-univoc transformation between $N$-representation and $\varphi$-representation.

In this case, the entropies in two candidates wave functions are the entropies, suffocating each other exponentially to the extremal point, the entropies are 
chained with interaction making the birth of matrix elements, which are the first term of the states density development.

For the normalized current density matrix elements, we write:

$$
\begin{aligned}
& \frac{d|\Psi|^{2}}{d t}=-\left|d i v_{\alpha} d i v_{\beta} j_{\alpha \beta}\right|^{\frac{1}{2}}, \\
& j_{\alpha \beta}=\int \Psi_{\alpha}(A) \hat{J} \Psi_{\beta}(A) d A,
\end{aligned}
$$

where $d A$ is the differential of the potential.

The operatorial transcription of the macroscopicity giving the distance between levels, we write

$$
\hat{e}^{S_{0}} \Delta F=S_{n 0} j_{\alpha \beta},
$$

then we obtain

$\hat{e}^{S_{0}}\left(\left|i \hbar \nabla+\frac{e^{*}}{c} A\right| \Psi\right)=-A \int\left|d i v_{\alpha} d i v_{\beta} j_{\alpha \beta}\right|^{\frac{1}{2}} d t+S_{n 0} j_{\alpha \beta}$.

By concerning the similitude laws for the second order transition, the action of $\hat{e}^{S_{0}}$ operator is equalling $\frac{b}{2}|\Psi|^{4}$ with $S_{n 0} j_{\alpha \beta}$, physically it signifies that, for an eigenvalue $S_{0}$ of the reduced entropy corresponding to the $j_{\alpha \beta}$ element, the term $|\Psi|^{4}$ is not mentioning a spatial extension except those permitted by the multiplier of $\frac{b}{2}$. The constant multiplication here is the $|\Psi|^{4}$ length scale coherence normalization.

In our work, we have considered the nullity of entropy as an asymptotic behaviour of the entropies wave functions, because of its definition as represented by $\hat{e}^{S_{0}}$ with conjugate $\hat{e}^{-S_{0}}$. The computing origin of the reduced entropy, will began by a negative number which is $-5.0396 \times 10^{-15}$.

\section{The entropy thermodynamical scale}

We shall show, at less, as a principle that it is possible to construct a thermodynamical scales of entropies, having one of the temperatures, with the fundamental definition $S=\int \frac{1}{T} d E+c t e$, by defining a reduced temperature and identifying these two equations: $S_{0}=S_{\alpha \beta}$ and $S=\int \frac{1}{T} d E+c t e$, we obtain
$S_{\alpha \beta}=\int \frac{1}{T} d E-5.0396 \cdot 10^{-15}$

Let us write the eigenvalue of reduced entropy as $\hat{e}^{S_{0}} \Delta F=S_{n 0} j_{\alpha \beta}$, where $\Delta F$ is in the order of $(\overline{\Delta F})_{\alpha \beta}$, also the two eigenvalues as $\hat{e}^{S_{0}} \Delta F=S_{10} j_{\alpha \beta}$ and $\hat{e}^{S_{0}} \Delta F=S_{20} j_{\alpha \beta}$, which leads to

$\hat{e}^{S_{0}} \Delta F=\frac{1}{2}\left|S_{10} j_{\alpha \beta}-S_{20} j_{\alpha \beta}\right|$.

According to the general rule of operators derivation, we have

$$
\frac{\partial \hat{e}^{S_{0}}}{\partial t}=\frac{i}{\hbar}\left[\hat{H} \hat{e}^{S_{0}}-\hat{e}^{S_{0}} \hat{H}\right] .
$$

If $S_{10}=S_{20}=H, \quad$ we must have $\frac{i}{2 \hbar}\left|S_{10} j_{\alpha \beta}-S_{20} j_{\alpha \beta}\right|$, then

$\frac{\partial \hat{e}^{S_{0}}}{\partial t}=\frac{i}{2 \hbar}\left|S_{10} j_{\alpha \beta}-S_{20} j_{\alpha \beta}\right|$.

For the thermal equilibrium of second order realization, it is necessary that the reduced entropy matrix must be diagonalizable per pavement, we write

$S_{\alpha \beta}=\left\{\begin{array}{l}S_{\alpha}=S_{0} \quad \text { for } \delta \int \xi(T, \hat{T}, \tau) d \tau=0 \\ \overline{S_{\alpha \beta}}=S_{0} \text { for } \delta \int \xi(T, \hat{T}, \tau) d \tau \sim-5.0396 \cdot 10^{-15}\end{array}\right.$

It seems that the variation of coherence scale causes the birth of reduced entropy and, reversibly, $\delta \int \xi(T, \hat{T}, \tau) d \tau$ is equivalent to measurement process giving the eigenvalue of reduced entropy, we say that $\delta \int \xi(T, \hat{T}, \tau) d \tau$ has a lot of extremals.

\section{Thermal equilibrium equation}

Per pavement, where is defined a fluctuating order parameter, respecting the operators differentiation rule, we rewrite

$\frac{d \hat{e}^{S_{0}}}{d t} \Delta F=\frac{i}{\hbar}\left[\hat{e}^{S_{0}} \hat{H}-\hat{H} \hat{e}^{S_{0}}\right] \Delta F$,

$\frac{d \hat{e}^{S_{0}}}{d t} \Delta F=\frac{i}{\hbar}\left(\hat{e}^{S_{0}} \hat{H}\right) \Delta F-\frac{i}{\hbar}\left(\hat{H} \hat{e}^{S_{0}}\right) \Delta F=$ 
$=\frac{i}{\hbar} \hat{e}^{S_{0}}(\hat{H} \Delta F)-\frac{i}{\hbar} \hat{H}\left(\hat{e}^{S_{0}} \Delta F\right)$,

$\left(\frac{d}{d t} \hat{e}^{S_{0}}\right) \Delta F=\frac{i}{\hbar} \hat{e}^{S_{0}}(\hat{H} \Delta F)-\frac{i}{\hbar} \hat{H} S_{n 0} j_{\alpha \beta}$,

then we obtain the two equations of thermal equilibrium

$\left(\frac{d \hat{e}^{S_{0}}}{d t}-\frac{i}{\hbar} \hat{e}^{S_{0}} \hat{H}\right) \Delta F=-\frac{i}{\hbar} \hat{H} S_{n 0} j_{\alpha \beta}$.

In modulus,

$\left\{\left|\frac{d}{d t} \hat{e}^{S_{0}}-2\left(\frac{d}{d t} \hat{e}^{S_{0}}\right)\left(-\frac{i}{\hbar} \hat{e}^{S_{0}} \hat{H}\right)+\right| \frac{i}{\hbar} \hat{e}^{S_{0}} \hat{H}||\right\}(\Delta F)^{2}=$

$=-\frac{1}{\hbar^{2}}\left(\hat{H} S_{n 0} j_{\alpha \beta}\right)^{2}$.

\section{Conclusion concerning the thermal equilibrium representation}

To establish equation for thermal equilibrium by expressing the action of operator $\frac{i}{\hbar}\left[\frac{d \hat{e}^{S_{0}}}{d t}-\frac{i}{\hbar} \hat{e}^{S_{0}} \hat{H}\right]$ on $\Delta F$, this action permits the birth of current density matrix elements.

This kind of identity is an equivalence between two operatorial writings, this equivalence concern the effect and not the cause.

To establish a thermodynamical scale of entropies, this will be possible by expressing the normalized extremums of $\int_{\tau_{1}}^{\tau_{2}} \xi(T, \hat{T}, \tau) d \tau . \quad \tau$ expresses the normalized thermodynamical scales, obtained as an equivalence between $\frac{d \ln T}{d \tau}$ and the ratios

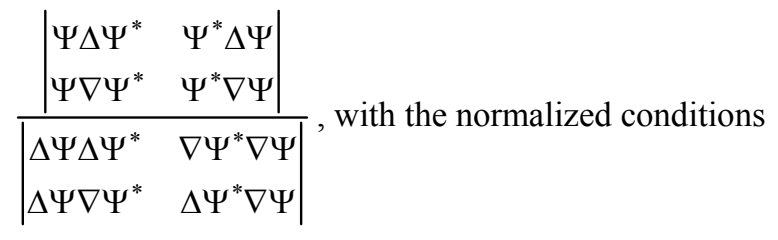

giving the equivalence between

$\int \Delta \Psi \hat{J} \Delta \Psi^{*} d q d q^{\prime}$

and $\iint \Delta \Psi \Delta \Psi^{*} \Delta \tau \hat{e}^{S_{0}} \Delta \Psi^{*} \Delta \Psi \Delta \tau^{\prime} d \tau d \tau^{\prime}$,

$\int \nabla \Psi \hat{J} \nabla \Psi^{*} d q d q^{\prime}$

and $\iint \nabla \Psi \nabla \Psi^{*} \Delta \tau \hat{e}^{S_{0}} \nabla \Psi^{*} \nabla \Psi \Delta \tau^{\prime} d \tau d \tau^{\prime}$,

$\int\left|\begin{array}{cc}\Delta \Psi & \nabla \Psi^{*} \\ \nabla \Psi & \Delta \Psi^{*}\end{array}\right|\left(j_{\alpha \beta} j_{\beta \gamma}-j_{\beta \gamma} j_{\alpha \beta}\right) d q^{2} d q^{\prime 2}$

and $\iint \nabla \Psi \nabla \Psi^{*} \Delta \tau \frac{i}{\hbar}\left[\hat{e}^{S_{0}} \hat{H}-\hat{H} \hat{e}^{S_{0}}\right] \Delta \Psi \Delta \Psi^{*} \Delta \tau^{\prime} d \tau d \tau^{\prime}$.

The coefficients of those equivalences are considered as the levels of normalizations.

\section{General conclusion}

The recursivity based on the J.H. Poincarre per pavement concept is permitting to introduce a limitation against the character of measurement processes, and the nature of values, emerging as a result of measurement without measurement, meaning by this that the reduced displaying levels of systems in nature appears as a result of interaction between two displaying faculties observed under two different indetermination orders $[1,2]$.

\section{References}

1. L. Landau, E. Lifshits, Statistical physics. Edition MIR, Moscow, 1969.

2. L. Landau, E. Lifshits, Quantum mechanics. Edition MIR, Moscow, 1967. 